\title{
Protein-protein Interfaces Integrated into Interaction Networks: Implications on Drug Design
}

\author{
Gozde Kar, Guray Kuzu, Ozlem Keskin and Attila Gursoy
}

Center for Computational Biology and Bioinformatics and College of Engineering, Koc University, Rumelifeneri Yolu, 34450 Sariyer Istanbul, Turkey

\begin{abstract}
The growing perception that diseases are often consequences of multiple molecular abnormalities rather than being the result of a single defect highlights the importance of network-centric view in therapeutic approaches. Protein interaction networks may contribute to understanding of disease, assist in drug design and discovery. Here, we review some recent advances in disease-associated protein interaction networks taking a structural approach. We first describe structural aspects of protein-protein interactions and properties of protein interfaces as related to drug design; we address protein interactions in a network perspective; in particular, we illustrate how integrating protein interfaces onto interaction networks can guide the identification of selective drug targets or drugs targeting multiple proteins in a network.
\end{abstract}

Keywords: Protein-protein interfaces, protein interaction networks, drug design.

\section{INTRODUCTION}

Protein-protein interactions have a key role in regulating many biological processes, cellular and signaling pathways. The dysfunction of these pathways due to the alterations in protein-protein interactions may lead to several diseases such as cancer and neurological disorders. Therefore, protein-protein interactions are widely considered as drug targets in disease states [1-3]. However, more recently, network-based approaches have gained importance in drug discovery with the comprehension that diseases are complex; a disease phenotype reflects several pathobiological processes that interact in a complex network [4]. The high interconnectivity in disease-associated networks suggests that it is better to target entire cellular pathways rather than single proteins [5].

Another point that makes the network-level endeavors valuable is the so-called polypharmacology, which has emerged as a new paradigm in drug discovery stating that one drug is more likely to bind to multiple distinct targets [6]. Following on from this principle, a drug may function in several different pathways; thus while being relevant in simultaneous treatment of several diseases, it might also create undesired side-effects. Earlier works suggest that even weak bindings to multiple targets may have profound effect on the biological system [7-9]. To understand such phenotypic responses, it is crucial to investigate the binding behavior of drugs. Drugs targeting protein-protein interactions, ultimately head protein interfaces where two protein chains come into contact. Consequently, understanding the details and principles of protein interfaces is immensely essential to develop a better strategy in drug design $[1,10]$. Obviously, one step further is to combine such structural details with the interaction networks to improve drug design concept; and that is the primary focus of this review.

Here, we first describe the physical and chemical aspects of protein-protein interactions important for drug design; focusing on hot spots, pockets, cavities at protein interfaces. Next, we review some recent network-level approaches in disease, drug design and discovery. We discuss the advantages of mapping protein interface structures into interaction networks, specifically in drug repurposing and predicting side-effects. Finally, we provide examples of therapeutic implications on drug design.

*Address correspondence to this author at the Center for Computational Biology and Bioinformatics and College of Engineering, Koc University, Rumelifeneri Yolu, 34450 Sariyer Istanbul, Turkey; Emails: gkar@ku.edu.tr; gkuzu@ku.edu.tr; okeskin@ku.edu.tr; agursoy@ku.edu.tr

\section{PHYSICAL AND CHEMICAL ASPECTS OF PROTEIN- PROTEIN INTERACTIONS IMPORTANT FOR DRUG DE- SIGN}

Biological reactions occur through the proteins, and the interactions among them play a crucial role. Protein interactions are biophysical phenomena. Although the medium is full of numerous molecules at different sizes, proteins to be interacted find each other and the interaction occurs. Proteins are interacting through their surfaces with the help of the shape and biochemical complementarity regarding the flexibility of the molecules and the environmental conditions [11-15]. Hence, protein interactions are special reactions rather than random processes.

\section{Interaction Occurs Through the Interface}

The region where the two molecules are contacting is called the binding site, or considering both sides, the interface. Understanding the protein structures is very important to identify these regions. If the structures of contacting proteins are known, it is quite easy to determine the interface. Interfacial residues are usually found by calculation of close residues from two sides based on the distance in the three dimensional space [16-18] or with the help of accessible surface area calculations [18-20].

Many studies investigate properties of interactions to enlighten protein interaction phenomena [13, 14, 16, 19-31]. The stability of the interaction is provided with binding forces; hydrogen bonds, salt bridges, electrostatic interactions and hydrophobic attractions $[32,33]$. Disulphide bonds are also but rarely seen between binding proteins. These attractions determine also the specificity of the interaction [33]. For example, obligate complexes rely usually on hydrophobic attractions and transient complexes on hydrogen bonds and salt bridges [13, 19, 21,33] although obligate interfaces are not more hydrophobic than transient ones [34, 35]. Understanding such details of the complexes is crucial in drug design; many signaling events, which are known to be disease-related, are regulated by transient protein-protein interactions [36, 37].

Considering the composition of the residues, we know that they usually differ between obligate and transient, or homo- and heterocomplexes [13, 19, 21, 33, 38-42]. Hydrophobic residues like Methionine, Phenylalanine, Proline, Alanine, Aspartic acid and Leucine (but not Isoleucine and Glycine) are commonly found in homodimer interfaces; whereas hydrophilic residues like Tryptophan, Cysteine, Histidine, Glutamine, Asparagine, Tyrosine and 
Serine (but not Threonine) are dominant in heterodimer interfaces $[21,42]$.

Interfaces are structurally similar to the cores of globular proteins [43], and many studies have shown that protein binding process is very similar to the protein folding [26-28, 44]. The structural shape of interfaces is planar and well packed, but also differs with respect to type of the interaction $[19,45]$. For example, relatively large surface areas are observed in homodimer complexes compared to heterodimer complexes [21, 42].

\section{Structural Features of Interfaces}

Proteins interact biophysically. Therefore, fundamental determinants in protein interactions are the shape and physicochemical complementarity $[11,13,46,47]$. Protein surfaces are full of pockets, crevices and indentations [48-51]. Some pockets which usually exist before binding are filled with the complementary protein like the key and lock model, when they associate during the interaction [52]. This is also valid for protein-ligand interactions. Size and shape of pockets are principally considered to design molecules or peptides like drugs. Therefore, structural information of the protein has a great importance in drug design. The number of small pockets on the protein surface is not very few $[16,19,21]$, on the contrary, much more than expected before. Besides, a cavity that does not pre-exist can be formed upon interacting with a small molecule [53]. In the lack of cavities, it becomes more difficult to inhibit a protein-protein interaction with a small molecule due to the flat large surface area.

In addition to the shape and size of the cavity, physicochemical properties of residues as well as their distribution in the cavity are important for the interaction. Thus, biochemical or electrochemical complementarity should also be considered in drug design. For example, due to the hydrophobicity of the interface, a drug to inhibit the protein-protein interaction should be designed to be hydrophobic [1, 54-57]. But this property makes the drug less soluble, less cell permeable and less selective for its targets [54-56].

Another parameter to characterize the interface is amino acid frequency $[40,58,59]$. The amino acid propensity of the interface is similar to interior of the protein $[23,60]$. The interface is usually rich of aromatic and hydrophilic residues, like Cysteine, Tyrosine, Phenylalanine and Tryptophan [23, 61]. Positively charged Arginine and Histidine also prefer to be at interface compared to both surface and core [62]. Threonine, Proline, Lysine, Glutamic Acid and Alanine are least commonly found in the interface [63]. Ofran and Rost [40] have predicted the type of the interaction with 63$100 \%$ accuracy rate just by using amino acid composition and residue-contact preferences. In another study, frequency of amino acids in transient and obligate complexes is analyzed. It is found that Glycine is more frequently seen in transient interfaces rather than the surfaces; whereas Glycine frequency is the same in interface and on surface of obligate complexes [61]. In the same study, pairwise contact preferences of the amino acid types are also investigated. Cystine-Cystine shows the highest preference of all possible contacts. This can be due to its ability to form disulfide bond. It is also found that this pair is more frequent in obligate interfaces than in transient interfaces. Moreover, acidic and basic amino acids are observed to be contacted mostly with other types of amino acids, but rarely with other amino acids with similar physicochemical properties. However, Histidine is found as an exception. It prefers to contact with itself but not with acidic amino acids. Furthermore, nonpolar amino acids prefer to contact with other nonpolar amino acids.

The other steric property of protein-protein interfaces is that they generally have an accessible surface area in the range of 1200$2000 \AA^{2}[13,64]$. This property is commonly used to distinguish biological interfaces from non-biological ones. Non-biological interfaces are formed between proteins which do not come together and contact each other actually in their physiological states [65].
Non-biological interactions are also called as crystal packing. These proteins are experimentally crystallized together; but it is mostly due to the enforcement by the crystallographic packing environment in the experiment. Non-biological interactions mostly have smaller accessible surface areas, which are about 400-600 $\AA^{2}[13$, 66]. However, it is not trivial to distinguish biological and nonbiological interactions just by considering the buried surface area. There are some examples of non-biological interactions with larger surface areas, which can be more than $2000 \AA^{2}$ [67-70], and some biological interactions, like protein-small molecule interactions (300-1000 $\AA^{2}$ ), have smaller contact areas [71, 72]. Therefore, computational approaches to predict biological interactions utilize a combination of interface properties, like surface area, residue conservation and amino acid composition [73-77]. Although significant success has been obtained in identification of biological interfaces or distinguishing biological and non-biological interactions, distinct properties of protein interfaces have not been certainly identified yet $[21,53,65]$.

\section{Interfaces are Conserved Throughout the Evolution}

Interacting proteins find each other specifically and selectively in a crowded medium. Therefore, proteins should identify each other through complementary interaction sites. This requires the conservation of the same (or similar) residues in the right orientation for both partners of the interaction [61]. To preserve the interaction, residues in the active or binding sides of proteins resist undergoing a change through the evolution $[78,79]$. It is proved in many studies that amino acids in interfaces are more conserved than on rest of the protein surfaces [61, 73, 80-87]. Conservation analysis of residues can be used to predict protein binding sites. The analyses can be based on the sequence $[3,88,89]$, or the structure $[87,90,91]$, or both sequence and structure [88, 92, 93]. When obligate and transient complexes are compared, obligate complexes are found to have more conserved interfaces than the transient complexes $[61,86]$. This supports that proteins of obligate complexes co-evolve, or proteins of transient complexes have higher ability to adapt to a change in their partners [86]. Moreover, analyses in the contact preferences of residues show that, residues prefer to contact with the other residues having a similar conservation grade [61]. In other words; highly conserved residues prefer to contact with highly conserved residues, and much variable residues prefer to contact with variable residues on the other side. Conserved residues are not randomly distributed and thus, some residues are more crucial than the others for the interaction [94]. If a contacting residue pair has an important role for the interaction, the residues are both conserved; if not, the residues are variable at the same grade [61].

The structures of interfaces are more conserved than the global structures of the proteins [95-97]. Even if their global structures and functions are different, proteins can interact through interfaces with similar architectures $[16,98]$. Structures of the interfaces have been clustered based on the domain or the whole structure. The Conserved Domain Database (CDD) provides protein domains conserved in evolution, and they are extracted via multiple sequence alignments [99, 100]. Schroeder and coworkers [101] have found 6,000 distinct types of interfaces by clustering domain interfaces. Sali and his group [102] have shown that proteins in the same SCOP families have similar binding architecture. Aloy and coworkers [103] have classified domain-based interactions of known threedimensional structure and developed 3 did web server. PPiClust provides clusters of similar 3D interface patterns in protein complexes [104]. Gao and Skolnick have found that structural space of protein-protein interfaces is close to complete and clustered interfaces of dimers into roughly 1,000 distinct types [105].

A structurally non-redundant dataset of protein-protein interfaces can be defined as three groups: Type I, Type II and Type III $[16,106]$. Type $I$ is the most common one. It includes interacting 
proteins with similar global structures. Type II contains proteins with similar interfaces but their global structures and functions are different. These structures are examples for the conservation of interface motifs even in the absence of global structural similarity $[45,107]$. In Type III, only one side of the interface is similar and the other side is somewhat different. Hub proteins are mostly examples of this type.

\section{Some Residues are Energetically More Important in the Inter- face: Hot Spots}

All contacting residues do not equally contribute to the binding energy. Regardless of the size of the binding site, only a few residues are responsible for the majority of the total binding energy [94, 108-110]. These residues are called "hot spots". Hot spots can be identified experimentally by Alanine Scanning Mutagenesis [81, 108]. In this method, each residue is mutated to an Alanine; and if a significant drop is observed in the energy due to the mutation, the residue is assigned as a hot spot. Hot spots can have different physicochemical properties; for example, they can be hydrophobic or polar [53, 86, 111]. Arginine, Tyrosine and Tryptophan are frequently found as hot spots; whereas, Leucine, Serine, Threonine, Valine [109, 112] are rarely. Methionine is rarely [109] or frequently [86] found as hot spot by different studies.

Hot spots are buried and tightly packed in the three dimensional space [94]. They are located near the center of the interface and away from the solvent $[10,109]$. However, they are usually found in discontinuous, discrete highly packed regions in interfaces. These clusters are called "hot regions" [94]. Furthermore, there is a strong correlation between structurally conserved residues and hot spots [86, 94, 113-115]. There are many computational studies based on their physical, biological and/or evolutionary features for the hot spot prediction. These approaches analyze combinations of hot spot features, like conservation, physicochemical properties, residue propensity, sequence profiles, accessible surface area, and contribution to binding energy [3, 92, 116-122]. Hot spots are also significant for drug design [123]. Since they are responsible for key contact potentials of the interactions, drugs are aimed to target these residues [10, 124-127]. The importance of hotspot residues in drug binding is exemplified in (Fig. 1): the antagonist L-685,818 targets to protein-protein interface of FKBP12-T beta R-I complex and binds to FKBP12 through its hot spot residues (Fig. 1C) and hence prevents its binding to T beta R-I (Fig. 1B).

\section{PROTEIN INTERACTION NETWORKS IN DISEASE AND DRUG DISCOVERY}

With the increase in availability of human protein interaction data [128], considerable attention has been directed towards studying the networks related to human disease [129]. In particular, protein interaction networks are inferred to investigate the topological properties of disease-associated genes. Wachi et al [130], for the first time, studied the human interaction map for the analysis of

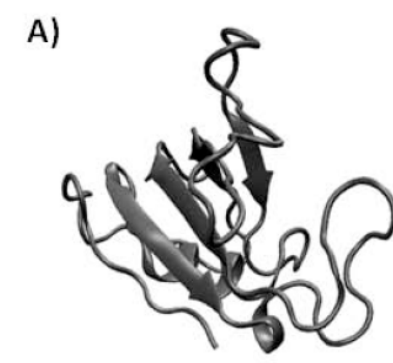

FKBP12 (2ppn)
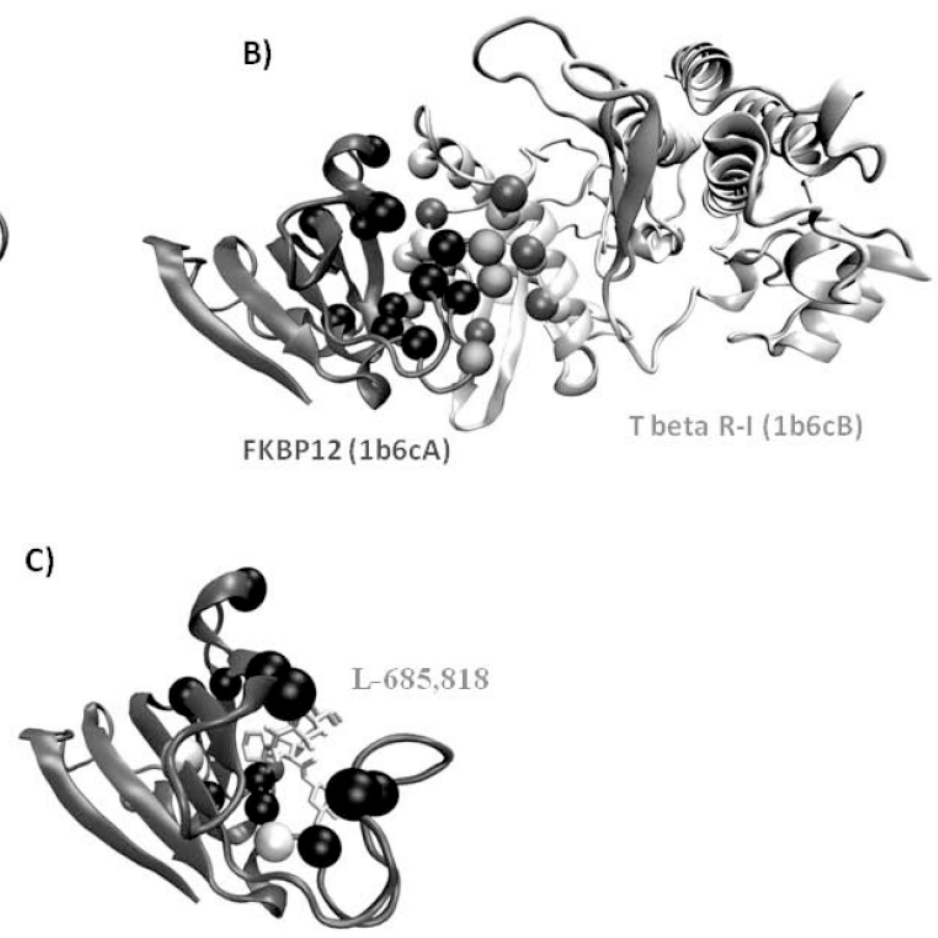

FKBP12 (1fkd)

Fig. (1). Molecular structure of FKBP12 protein alone, interacting with T beta R-I and the ligand L-685,818. (A) FKBP12 protein alone (PDB code: 2ppn). (B) The complex of FKBP12/T beta R-I (PDB code: 1b6c) and interacting residues. Computational hot spots of the interaction are identified using HotPoint server [171]: chain A: 26Y, 36F, 37D, 42R, 46F, 55V, 56I, 59W, 82Y, 87H, 90I, 91I; chain B: 184M, 195L, 196L, 197V, 199R, 242W, 246A, 250Q, 265A. Gray structure: FKBP12, Light gray structure: T beta R-I, Black balls: C-alpha of Hot spots on FKBP12, Gray balls: C-alpha of Non-hot spots on FKBP12, Light gray Balls: C-alpha of Hot spots on T beta R-I, Dark gray balls: C-alpha of Non-hot spots on T beta R-I. (C) The complex of FKBP12/ L-685,818 and interacting residues (PDB code: 1fkd). Gray structure: FKBP12, Light gray structure: L-685,818, Black balls: C-alpha of Hot spots on FKBP12 while interacting with T beta R-I, Gray ball: C-alpha of Non-hot spots on FKBP12 while interacting with T beta R-I, Light gray Balls: C-alpha of FKBP12 which do not interact with any atom of T beta R-I in the complex of FKBP12/T beta R-I. The ligand is interacting through 11 of 12 computational hot spots of the complex of FKBP12/T beta R-I (not interacting with 90I of chain $\mathbf{A})$. 
cancer and found that squamous cell lung cancer genes are highly connected and central, hence, display the same topological properties of essential genes. In another study, cancer proteins were found to have, on average, twice as many interaction partners as noncancer proteins [131]. Moving beyond cancer, in a study of human inherited ataxia disease, most of the ataxia-associated proteins were shown to form an interconnected sub-network in the protein interaction network suggesting that these proteins may function in a cooperative manner [132]. Considering the disease-associated proteins in OMIM Morbid Map [133], it was shown that they have more protein-protein interactions than do non-disease proteins [134]. Goh et al. [135] constructed the first human disease network by linking disease that share disease-associated genes and differently from previous studies, demonstrated that most disease-associated genes are non-essential and are not encoded by hub proteins. In addition to identifying topological properties of disease-associated genes, network-based approaches are also assessed to predict new potential disease-associated genes (see [4] for a recent review).

As aforementioned, a disease state is often the result of a complex combination of molecular events, which consequently implies the complexity of therapeutic approaches. In addition, biological systems are robust; they can often bypass a perturbation and maintain their functions through different mechanisms such as back-up circuits and fail-safe mechanisms [136]. Hence, when selecting the putative drug targets, it is crucial to consider their positioning in the network [5]. Analysis of the network properties of known human drug targets showed that they tend to occur at middle-degree to low-degree nodes, i.e. less connected nodes in protein-protein interaction network [137]. This finding implies that targeting less prominent nodes in a protein interaction network can create fewer side effects and increase synergetic efficacy for combinatorial drugs [137]. Ranking the proteins by topological properties in the human protein-protein interaction network was also shown to be useful in drug-target identification [138]. Another approach, which seems to make major contributions to drug discovery lately, is to include structural details of proteins into networks and discussed in the following section.

Protein-protein interaction databases provide an invaluable resource to study protein-protein interactions at a network level. These databases grow rapidly as the number of interactiondetection experiments, genome sequencing experiments and proteins with solved structures increase. Among these, the Database of Interacting Proteins (DIP) [139], the Biomolecular Interaction Network Database (BIND - converted to Proteomics Standard Initiative-Molecular Interaction, PSI-MI 2.5) [140], the BioGRID General Repository for Interaction Datasets [141], IntAct [142], the Human Protein Reference Database (HPRD) [143], the Munich Information Center for Protein Sequences (MIPS) [144], the Human Protein Interaction Database (HPID) [145], the database of Protein Structural Interactome map (PSIbase) [146] and the database of Protein Domain Interactions (DOMINE) [147] list experimentally determined protein-protein interactions. From these, PSIbase and DOMINE include the binding site information, i.e. they indicate where two proteins interact and hence can assist in structural studies in disease.

\section{INCLUDING PROTEIN STRUCTURAL INFORMATION INTO INTERACTION NETWORKS: ADVANTAGES IN DRUG DESIGN}

The pioneering work of Aloy and Russell [148] illustrated how three dimensional protein structures can be used to infer molecular details of interactions in a network. Following this, structured networks are utilized to understand diseases. Dawelbait et al. [149] constructed a network related to pancreatic cancer by combining known interactions and structure-based interaction predictions. They predicted 40 novel interactions that are specific to pancreatic cancer. In one study, cancer-associated signaling pathways and their physical protein-protein interactions are analyzed with the goal of providing insights into three-dimensional structure-function relationship [150]. In another study, protein interface structures are integrated to human cancer protein interaction network and interface analysis revealed that cancer-related proteins have smaller, more planar, more charged and less hydrophobic binding sites than non-cancer proteins, which may indicate low affinity and high specificity of the cancer-related interactions [151]. Additionally, integrating interfaces into networks can provide timing of proteins' interactions; whether they are simultaneous or exclusive [152-154].

Besides inferring the nature of disease-related interactions and their role in the network, comparison of protein-protein interfaces across an interactome can also assist in identifying drug targets or drugs targeting multiple proteins to block parallel pathways in a network [155]. With structural analysis drug binding pockets can be identified and compared with binding pockets of other proteins in the network, which could eventually lead to discovering candidates for drug-targetable protein-protein interactions [156]. Since the number of distinct binding motifs is limited in nature [157], structurally different proteins can share similar interface architectures [16]. Therefore, even though two proteins are structurally different and belong to different pathways in the interactome, if their binding pockets are similar, a drug can bind to both. While this is possible, binding also depends on the chemical nature of the drug ligand itself [158]. It was proposed that the increase in charged residues in a designed peptide drug can provide conformational flexibilities and enhance the propensity to bind multiple proteins [155].

Although there occurs some limitations such as incompleteness of protein interaction networks and structural data, combining protein interfaces with interaction networks could guide identification of all target proteins that are influenced by a drug, either positively or negatively. Considering the position and role of these target proteins in the network could help to predict side-effects or to discover a treatment of a new disease. The latter is called drug repurposing, which means finding new uses of old drugs [159], is gradually gaining popularity since de novo drug design is laborious and very costly [160]. One example is entacapone, which targets catechol-Omethyltransferase and is used in the treatment of Parkinson's disease [161]. Based on binding site similarity, Kinnings et al. [161] identified a new target of entacapon: enoyl-acyl carrier protein reductase. This new target, which is important in the fatty acid biosynthesis in tuberculosis, is experimentally verified [161] and inhibiting this protein by entacapone offers a way to treat infections such as tuberculosis. Another widely known example is the celecoxib (Celebrex), which is a nonsteroidal anti-inflammatory drug that is known to bind cyclooxygenase-2. Weber et al. [162] showed that celecoxib also binds to a totally unrelated protein; carbonic anhydrase, which has a structurally similar binding site to that of cyclooxygenase- 2 . This finding reveals a new role of celecoxib in the treatment of glaucoma and possibly for cancer [162]. Provided that protein structures and binding sites are known in a diseaseassociated network, predicting druggable proteins and the resulting effects will be less demanding. This concept is visualized in (Fig. 2) with the celecoxib example.

\section{CASE STUDY: A PROMISCUOUS DRUG: IMATINIB (GLEEVEC)}

The concept that one drug binding to multiple distinct targets [6] is known as polypharmacology or drug promiscuity. "One drugone target" paradigm erodes $[6,163]$ and several drugs are observed to be promiscuous [164]. One of them is imatinib (also known as Gleevec), which is a milestone in small-molecule drug discovery and is successful in cancer therapy [165]. Imatinib is first designed as a drug targeting Brc-Abl tyrosine kinase, which causes chronic myelogenous leukemia [166]. Later on, it is realized that imatinib is not entirely specific; it can bind to tyrosine kinases other than $\mathrm{Abl}$ such as KIT and PDGFR $\alpha$ [166]. Subsequently, imatinib has also 


\section{Disease-associated structural networks Drug targeting multiple proteins Drug binding site \&}

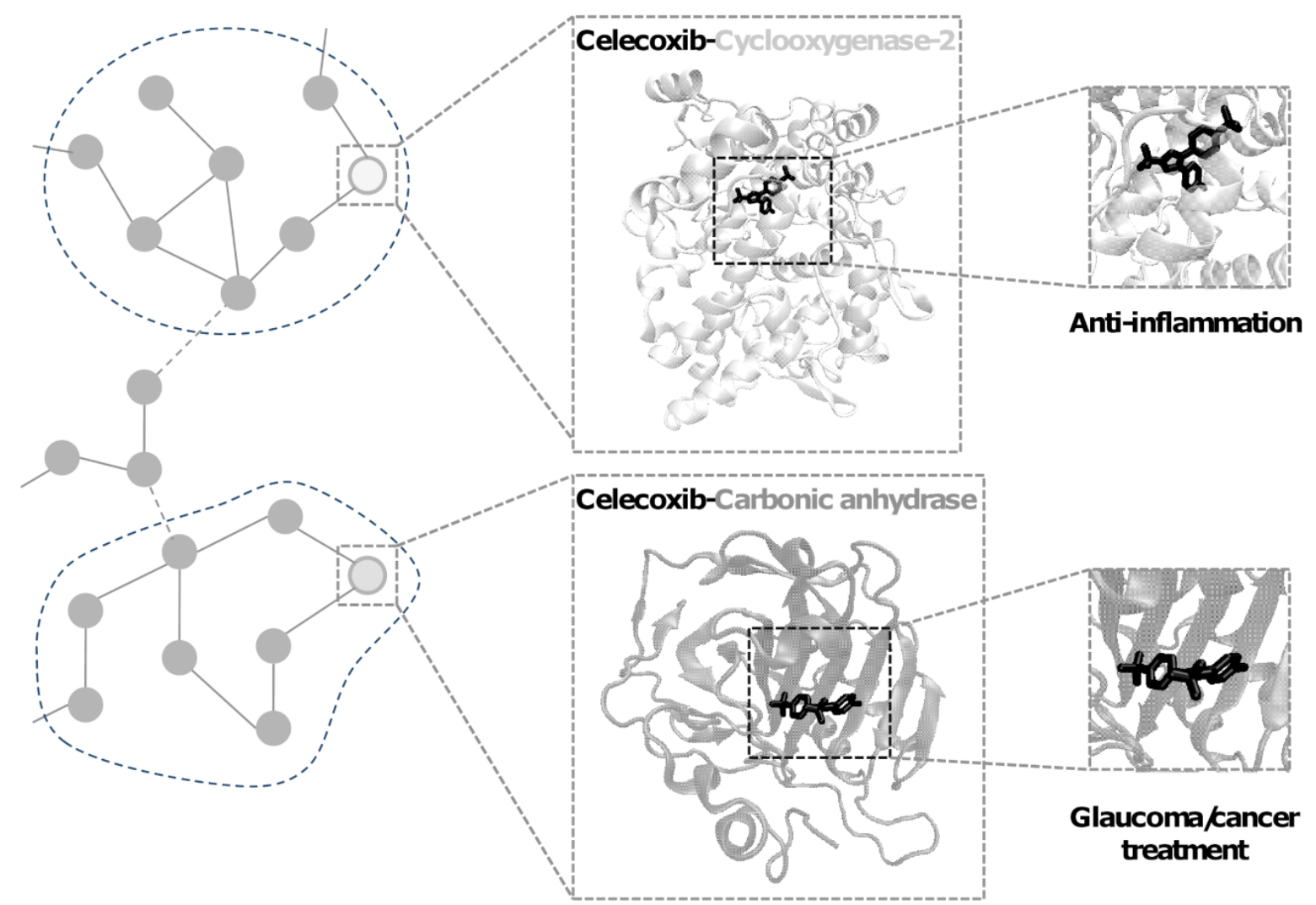

Fig. (2). Concept figure. Protein structures are integrated into disease-associated networks. Identifying protein interfaces and binding pockets in diseaseassociated structural network can assist in finding drug targets and role of these proteins in disease. As an example, a nonsteroidal anti-inflammatory drug, which is known to target multiple proteins, is visualized. It is interesting that although cyclooxygenase-2 (in white color, PDB code: $311 \mathrm{n}$ ) and carbonic anhydrase (in gray color, PDB code: 1oq5) are structurally different proteins; all-alpha and all-beta structure, respectively, their binding sites in contact with celecoxib share structural and physico-chemical similarities [162]. Latterly found interaction with carbonic anhydrase reveals the new role of celecoxib in the treatment of glaucoma and possibly for cancer [162].

been approved for the treatment of gastrointestinal stromal tumors, which is caused by mutations in KIT or PDGFR $\alpha$ [166]. Binding sites of Abl and KIT to imatinib are similar (Fig. 3). Imatinib seems to have potential for use in a variety of other diseases; for example, more recently, a non-kinase target; NQO2 is identified for imatinib [167] (Fig. 3). NQO2 is a cytoplasmic flavoprotein functioning in the cellular response to oxidative stress [167] and its inhibition by imatinib can lead to anti-inflammatory effects [165]. Comparison of the complex structures of imatinib with Abl and NQO2 shows that conformation of imatinib and binding nature differ: imatinib interacts with NQO2 through hydrophobic interactions without any hydrogen bonds, whereas it uses six hydrogen bonds and several van der Waals interactions in binding to Abl kinase [167, 168]. Imatinib adopts an extended conformation in binding to Abl and that is quite different from the compact conformation seen in the NQO2 structure. Consequently, to identify potential targets of a drug, its distinct conformations are also needed to be taken into account. Known side effects of imatinib include muscle cramps, edema, diarrhea and bone-narrow toxicity [166] although their cause is currently unclear. However, yielding adverse effects is an expected outcome since imatinib is more likely to have several yet unknown targets as well and appears to attack multiple pathways, such as cell cycle and many signaling pathways according to KEGG pathway database annotations [169]. Clearly, a network- centric approach with inclusion of structural and dynamic details will assist in understanding the biological and kinetic profile of promiscuous drugs.

\section{CONCLUSIONS}

Many promising drug candidates fail the last clinical phases since mechanisms of the disease pathways they target are yet unclear or off-target effects cannot be predicted by in-vitro models [5]. Network-centric approaches offer a way to focus on entire cellular pathways, and hence provide a global perspective in drug design. Protein three-dimensional structure information is further required to examine atomic details of protein-ligand interactions, in particular, to understand how proteins interact with drugs and the resulting functional effect of these interactions. Although structural coverage of the proteome is limited, the structures deposited in the Protein Data Bank has been increasing at a fast rate [170], which will assist in structural studies. Obviously, for drug design studies, finding and analyzing the binding sites of proteins can reveal important clues. We know that some residues (hot spots) in the interfaces are more critical than others to the stability of the complex. As exemplified in this study for the case of FKBP12, probably, a drug ligand is more likely to interact with its target protein utilizing the hot spot residues of the target. Since similar binding sites usually bind the same drug ligands, in a disease-associated structural 


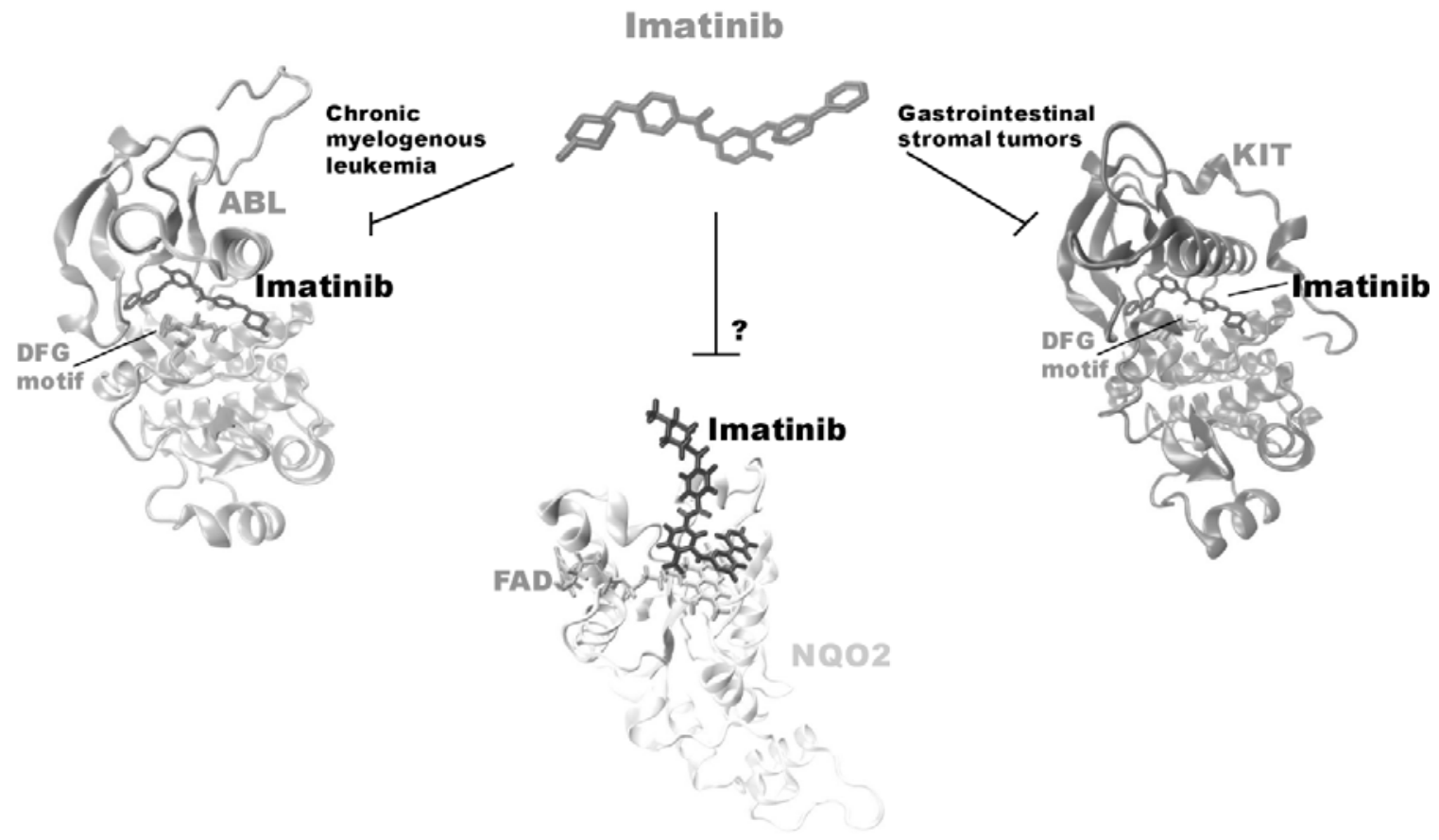

Fig. (3). A promiscuous drug; imatinib binding to different targets. Imanitib binds and inhibits ABL tyrosine kinase and hence is used in the treatment of chronic myelogenous leukemia (left panel). Imatinib also can bind to another kinase: KIT which has a similar binding site to that of ABL (mainly DFG motif is utilized through interacting with imatinib) (right panel). KIT is targeted by imatinib in the treatment of gastrointestinal stromal tumors. Latterly discovered non-kinase target of imatinib is NQO2 (the panel below) although the resulting effect of this interaction is not clear. In this case, imatinib adopts a different conformation, i.e. more compact, and binds to cofactor FAD of NQO2 making mostly hydrophobic contacts.

network, identifying which binding sites of which proteins show similarity could reveal possible targets of the drugs and ultimately, the possible positive or negative side effects.

\section{REFERENCES}

[1] Chene P. Drugs targeting protein-protein interactions. Chem Med Chem 2006; 1: 400-11.

[2] Fry DC. Protein-protein interactions as targets for small molecule drug discovery. Biopolymers 2006; 84: 535-52.

[3] Gonzalez-Ruiz D, Gohlke H. Targeting protein-protein interactions with small molecules: challenges and perspectives for computational binding epitope detection and ligand finding. Curr Med Chem 2006; 13: 2607-25.

[4] Barabasi AL, Gulbahce N, Loscalzo J. Network medicine: a network-based approach to human disease. Nat Rev Genet 2011; 12: 56-68.

[5] Pujol A, Mosca R, Farres J, Aloy P. Unveiling the role of network and systems biology in drug discovery. Trends Pharmacol Sci 2010; 31: 115-23.

[6] Hopkins AL. Network pharmacology: the next paradigm in drug discovery. Nat Chem Biol 2008; 4: 682-90.

[7] Agoston V, Csermely P, Pongor S. Multiple weak hits confuse complex systems: a transcriptional regulatory network as an example. Phys Rev E Stat Nonlin Soft Matter Phys 2005; 71: 051909.

[8] Badis G, Berger MF, Philippakis AA, et al. Diversity and complexity in DNA recognition by transcription factors. Science 2009; 324: 1720-3.

[9] Roy J, Cyert MS. Cracking the phosphatase code: docking interactions determine substrate specificity. Sci Signal 2009; 2: re9.

[10] Arkin MR, Wells JA. Small-molecule inhibitors of protein-protein interactions: progressing towards the dream. Nat Rev Drug Discov 2004; 3: 301-17.

[11] Grunberg R, Leckner J, Nilges M. Complementarity of structure ensembles in protein-protein binding. Structure 2004; 12: 2125-36.

[12] Lawrence MC, Colman PM. Shape complementarity at protein/protein interfaces. J Mol Biol 1993; 234: 946-50.
[13] Lo Conte L, Chothia C, Janin J. The atomic structure of proteinprotein recognition sites. J Mol Biol 1999; 285: 2177-98.

[14] Sheinerman FB, Norel R, Honig B. Electrostatic aspects of proteinprotein interactions. Curr Opin Struct Biol 2000; 10: 153-9.

[15] Zhang Q, Sanner M, Olson AJ. Shape complementarity of proteinprotein complexes at multiple resolutions. Proteins 2009; 75: 45367.

[16] Keskin O, Tsai CJ, Wolfson H, Nussinov R. A new, structurally nonredundant, diverse data set of protein-protein interfaces and its implications. Protein Sci 2004; 13: 1043-55.

[17] Gong S, Yoon G, Jang I, et al. PSIbase: a database of Protein Structural Interactome map (PSIMAP). Bioinformatics 2005; 21 2541-3.

[18] Gadkari RA, Varughese D, Srinivasan N. Recognition of interaction interface residues in low-resolution structures of protein assemblies solely from the positions of $\mathrm{C}$ (alpha) atoms. PLoS One 2009; 4: e4476.

[19] Jones S, Thornton JM. Principles of protein-protein interactions. Proc Natl Acad Sci USA 1996; 93: 13-20.

[20] Jones S, Marin A, Thornton JM. Protein domain interfaces: characterization and comparison with oligomeric protein interfaces. Protein Eng 2000; 13: 77-82.

[21] Jones S, Thornton JM. Analysis of protein-protein interaction sites using surface patches. J Mol Biol 1997; 272: 121-32.

[22] Keskin O, Ma B, Rogale K, Gunasekaran K, Nussinov R. Proteinprotein interactions: organization, cooperativity and mapping in a bottom-up Systems Biology approach. Phys Biol 2005; 2: S24-35.

[23] Chakrabarti P, Janin J. Dissecting protein-protein recognition sites. Proteins 2002; 47: 334-43.

[24] Chothia C, Janin J. Principles of protein-protein recognition. Nature 1975; 256: 705-8.

[25] Keskin O, Nussinov R. Favorable scaffolds: proteins with different sequence, structure and function may associate in similar ways. Protein Eng Des Sel 2005; 18: 11-24.

[26] Tsai CJ, Lin SL, Wolfson HJ, Nussinov R. Studies of proteinprotein interfaces: a statistical analysis of the hydrophobic effect. Protein Sci 1997; 6: 53-64. 
[27] Tsai CJ, Nussinov R. Hydrophobic folding units at protein-protein interfaces: implications to protein folding and to protein-protein association. Protein Sci 1997; 6: 1426-37.

[28] Tsai CJ, Xu D, Nussinov R. Structural motifs at protein-protein interfaces: protein cores versus two-state and three-state model complexes. Protein Sci 1997; 6: 1793-805.

[29] Negi SS, Braun W. Statistical analysis of physical-chemical properties and prediction of protein-protein interfaces. J Mol Model 2007; 13: 1157-67.

[30] Gruber J, Zawaira A, Saunders R, Barrett CP, Noble ME. Computational analyses of the surface properties of protein-protein interfaces. Acta Crystallogr D Biol Crystallogr 2007; 63: 50-7.

[31] Headd JJ, Ban YE, Brown P, et al. Protein-protein interfaces: properties, preferences, and projections. J Proteome Res 2007; 6: 2576-86.

[32] Xu D, Tsai CJ, Nussinov R. Hydrogen bonds and salt bridges across protein-protein interfaces. Protein Eng 1997; 10: 999-1012.

[33] De S, Krishnadev O, Srinivasan N, Rekha N. Interaction preferences across protein-protein interfaces of obligatory and nonobligatory components are different. BMC Struct Biol 2005; 5: 15.

[34] Teyra J, Pisabarro MT. Characterization of interfacial solvent in protein complexes and contribution of wet spots to the interface description. Proteins 2007; 67: 1087-95.

[35] Rodier F, Bahadur RP, Chakrabarti P, Janin J. Hydration of protein-protein interfaces. Proteins 2005; 60: 36-45.

[36] Kar G, Keskin O, Gursoy A, Nussinov R. Allostery and population shift in drug discovery. Curr Opin Pharmacol 2010; 10: 715-22.

[37] Ozbabacan SE, Engin HB, Gursoy A, Keskin O. Transient proteinprotein interactions. Protein Eng Des Sel 2011; 24: 635-48.

[38] Guharoy M, Chakrabarti P. Secondary structure based analysis and classification of biological interfaces: identification of binding motifs in protein-protein interactions. Bioinformatics 2007; 23: 1909-18.

[39] Mintseris J, Weng Z. Structure, function, and evolution of transient and obligate protein-protein interactions. Proc Natl Acad Sci USA 2005; 102: 10930-5.

[40] Ofran Y, Rost B. Analysing six types of protein-protein interfaces. J Mol Biol 2003; 325: 377-87.

[41] Vacic V, Uversky VN, Dunker AK, Lonardi S. Composition Profiler: a tool for discovery and visualization of amino acid composition differences. BMC Bioinformatics 2007; 8: 211.

[42] Zhanhua C, Gan JG, Lei L, Sakharkar MK, Kangueane P. Protein subunit interfaces: heterodimers versus homodimers. Bioinformation 2005; 1: 28-39.

[43] Tuncbag N, Gursoy A, Guney E, Nussinov R, Keskin O. Architectures and functional coverage of protein-protein interfaces. J Mol Biol 2008; 381: 785-802.

[44] Haliloglu T, Keskin O, Ma B, Nussinov R. How similar are protein folding and protein binding nuclei? Examination of vibrational motions of energy hot spots and conserved residues. Biophys $\mathbf{J}$ 2005; 88: 1552-9.

[45] Keskin O, Nussinov R. Similar binding sites and different partners: implications to shared proteins in cellular pathways. Structure 2007; 15: 341-54.

[46] Veselovsky AV, Ivanov YD, Ivanov AS, et al. Protein-protein interactions: mechanisms and modification by drugs. J Mol Recognit 2002; 15: 405-22.

[47] Tsuchiya Y, Nakamura H, Kinoshita K. Discrimination between biological interfaces and crystal-packing contacts. Advances and Applications in Bioinformatics and Chemistry 2008; 2008: 99 113.

[48] Kuhn D, Weskamp N, Schmitt S, Hullermeier E, Klebe G. From the similarity analysis of protein cavities to the functional classification of protein families using cavbase. J Mol Biol 2006; 359: 1023-44.

[49] Binkowski TA, Naghibzadeh S, Liang J. CASTp: Computed Atlas of Surface Topography of proteins. Nucleic Acids Res 2003; 31: 3352-5.

[50] Dundas J, Ouyang Z, Tseng J, et al. CASTp: computed atlas of surface topography of proteins with structural and topographical mapping of functionally annotated residues. Nucleic Acids Res 2006; 34: W116-8.

[51] An J, Totrov M, Abagyan R. Pocketome via comprehensive identification and classification of ligand binding envelopes. Mol Cell Proteomics 2005; 4: 752-61.
[52] Li X, Keskin O, Ma B, Nussinov R, Liang J. Protein-protein interactions: hot spots and structurally conserved residues often locate in complemented pockets that pre-organized in the unbound states: implications for docking. J Mol Biol 2004; 344: 781-95.

[53] Veselovsky AV, Archakov AI. Inhibitors of protein-protien interactions as potential drugs. Curr Comput Aided Drug Des 2007; 3: 51-8.

[54] Arkin M. Protein-protein interactions and cancer: small molecules going in for the kill. Curr Opin Chem Biol 2005; 9: 317-24.

[55] Lipinski CA, Lombardo F, Dominy BW, Feeney PJ. Experimental and computational approaches to estimate solubility and permeability in drug discovery and development settings. Adv Drug Deliv Rev 2001; 46: 3-26.

[56] Walters WP, Ajay, Murcko MA. Recognizing molecules with druglike properties. Curr Opin Chem Biol 1999; 3: 384-7.

[57] Bradford JR, Needham CJ, Bulpitt AJ, Westhead DR. Insights into protein-protein interfaces using a Bayesian network prediction method. J Mol Biol 2006; 362: 365-86.

[58] Larsen TA, Olson AJ, Goodsell DS. Morphology of protein-protein interfaces. Structure 1998; 6: 421-7.

[59] Nguyen MN, Rajapakse JC, Duan K. Amino acid features for prediction of protein-protein interface residues with support vector machines. Proceedings of EvoBIO 2007; 2007: 187 - 96.

[60] Zhanhua C, Gan JG, Lei L, et al. Identification of critical heterodimer protein interface parameters by multi-dimensional scaling in euclidian space. Front Biosci 2005; 10: 844-52.

[61] Von Eichborn J, Gunther S, Preissner R. Structural features and evolution of protein-protein interactions. Genome Inform 2010; 22: $1-10$.

[62] Baussand J, Camproux AC. Deciphering the shape and deformation of secondary structures through local conformation analysis. BMC Struct Biol 2011; 11: 9.

[63] Neuvirth H, Raz R, Schreiber G. ProMate: a structure based prediction program to identify the location of protein-protein binding sites. J Mol Biol 2004; 338: 181-99.

[64] Horton N, Lewis M. Calculation of the free energy of association for protein complexes. Protein Sci 1992; 1: 169-81.

[65] Tuncbag N, Kar G, Keskin O, Gursoy A, Nussinov R. A survey of available tools and web servers for analysis of protein-protein interactions and interfaces. Brief Bioinform 2009; 10: 217-32.

[66] Henrick K, Thornton JM. PQS: a protein quaternary structure file server. Trends Biochem Sci 1998; 23: 358-61.

[67] Janin J. Specific versus non-specific contacts in protein crystals. Nat Struct Biol 1997; 4: 973-4.

[68] Wodak SJ, Mendez R. Prediction of protein-protein interactions: the CAPRI experiment, its evaluation and implications. Curr Opin Struct Biol 2004; 14: 242-9.

[69] Norel R, Petrey D, Wolfson HJ, Nussinov R. Examination of shape complementarity in docking of unbound proteins. Proteins 1999; 36: 307-17.

[70] Gray JJ, Moughon S, Wang C, et al. Protein-protein docking with simultaneous optimization of rigid-body displacement and sidechain conformations. J Mol Biol 2003; 331: 281-99.

[71] Cheng AC, Coleman RG, Smyth KT, et al. Structure-based maximal affinity model predicts small-molecule druggability. Nat Biotechnol 2007; 25: 71-5.

[72] Smith RD, Hu L, Falkner JA, et al. Exploring protein-ligand recognition with Binding MOAD. J Mol Graph Model 2006; 24: 414-25.

[73] Valdar WS, Thornton JM. Conservation helps to identify biologically relevant crystal contacts. J Mol Biol 2001; 313: 399416.

[74] Zhu H, Domingues FS, Sommer I, Lengauer T. NOXclass: prediction of protein-protein interaction types. BMC Bioinformatics 2006; 7: 27.

[75] Bernauer J, Bahadur RP, Rodier F, Janin J, Poupon A. DiMoVo: a Voronoi tessellation-based method for discriminating crystallographic and biological protein-protein interactions. Bioinformatics 2008; 24: 652-8.

[76] Liu S, Li Q, Lai L. A combinatorial score to distinguish biological and nonbiological protein-protein interfaces. Proteins 2006; 64: 6878.

[77] Liu Q, Li J. Propensity vectors of low-ASA residue pairs in the distinction of protein interactions. Proteins 2010; 78: 589-602. 
[78] Shakhnovich BE, Dokholyan NV, DeLisi C, Shakhnovich EI. Functional fingerprints of folds: evidence for correlated structurefunction evolution. J Mol Biol 2003; 326: 1-9.

[79] Torrance JW, Bartlett GJ, Porter CT, Thornton JM. Using a library of structural templates to recognise catalytic sites and explore their evolution in homologous families. J Mol Biol 2005; 347: 565-81

[80] Caffrey DR, Somaroo S, Hughes JD, Mintseris J, Huang ES. Are protein-protein interfaces more conserved in sequence than the rest of the protein surface? Protein Sci 2004; 13: 190-202.

[81] Thorn KS, Bogan AA. ASEdb: a database of alanine mutations and their effects on the free energy of binding in protein interactions. Bioinformatics 2001; 17: 284-5.

[82] Pupko T, Bell RE, Mayrose I, Glaser F, Ben-Tal N. Rate4Site: an algorithmic tool for the identification of functional regions in proteins by surface mapping of evolutionary determinants within their homologues. Bioinformatics 2002; 18 Suppl 1: S71-7.

[83] Grishin NV, Phillips MA. The subunit interfaces of oligomeric enzymes are conserved to a similar extent to the overall protein sequences. Protein Sci 1994; 3: 2455-8.

[84] Ouzounis C, Perez-Irratxeta C, Sander C, Valencia A. Are binding residues conserved? Pac Symp Biocomput 1998; 401-12.

[85] Bartlett GJ, Porter CT, Borkakoti N, Thornton JM. Analysis of catalytic residues in enzyme active sites. J Mol Biol 2002; 324: 105-21.

[86] Ma B, Elkayam T, Wolfson H, Nussinov R. Protein-protein interactions: structurally conserved residues distinguish between binding sites and exposed protein surfaces. Proc Natl Acad Sci USA 2003; 100: 5772-7.

[87] Konc J, Janezic D. Protein-protein binding-sites prediction by protein surface structure conservation. J Chem Inf Model 2007; 47: 940-4.

[88] Watson JD, Laskowski RA, Thornton JM. Predicting protein function from sequence and structural data. Curr Opin Struct Biol 2005; 15: 275-84.

[89] Wang B, Ge LS, Huang D, Wong HS. Prediction of protein-protein interacting sites by combining SVM algorithm with Bayesian method. ICNC 2007 2007; 2: 329 - 33.

[90] Laskowski RA, Watson JD, Thornton JM. Protein function prediction using local 3D templates. J Mol Biol 2005; 351: 614-26.

[91] Laskowski RA, Watson JD, Thornton JM. ProFunc: a server for predicting protein function from 3D structure. Nucleic Acids Res 2005; 33: W89-93.

[92] Kortemme T, Baker D. A simple physical model for binding energy hot spots in protein-protein complexes. Proc Natl Acad Sci USA 2002; 99: 14116-21.

[93] Chung JL, Wang W, Bourne PE. Exploiting sequence and structure homologs to identify protein-protein binding sites. Proteins 2006; 62: 630-40.

[94] Keskin O, Ma B, Nussinov R. Hot regions in protein--protein interactions: the organization and contribution of structurally conserved hot spot residues. J Mol Biol 2005; 345: 1281-94.

[95] Illergard K, Ardell DH, Elofsson A. Structure is three to ten times more conserved than sequence--a study of structural response in protein cores. Proteins 2009; 77: 499-508

[96] Valas RE, Yang S, Bourne PE. Nothing about protein structure classification makes sense except in the light of evolution. Curr Opin Struct Biol 2009; 19: 329-34.

[97] Zhang QC, Petrey D, Norel R, Honig BH. Protein interface conservation across structure space. Proc Natl Acad Sci USA 2010; 107: 10896-901

[98] Tsai CJ, Lin SL, Wolfson HJ, Nussinov R. A dataset of proteinprotein interfaces generated with a sequence-order-independent comparison technique. J Mol Biol 1996; 260: 604-20.

[99] Marchler-Bauer A, Anderson JB, DeWeese-Scott C, et al. CDD: a curated Entrez database of conserved domain alignments. Nucleic Acids Res 2003; 31: 383-7.

[100] Marchler-Bauer A, Panchenko AR, Shoemaker BA, et al. CDD: a database of conserved domain alignments with links to domain three-dimensional structure. Nucleic Acids Res 2002; 30: 281-3.

[101] Kim WK, Henschel A, Winter C, Schroeder M. The many faces of protein-protein interactions: A compendium of interface geometry. PLoS Comput Biol 2006; 2: e124.

[102] Korkin D, Davis FP, Sali A. Localization of protein-binding sites within families of proteins. Protein Sci 2005; 14: 2350-60.
[103] Stein A, Ceol A, Aloy P. 3did: identification and classification of domain-based interactions of known three-dimensional structure. Nucleic Acids Res 2011; 39: D718-23.

[104] Aung Z, Tan SH, Ng SK, Tan KL. PPiClust: efficient clustering of 3D protein-protein interaction interfaces. J Bioinform Comput Biol 2008; 6: 415-33

[105] Gao M, Skolnick J. Structural space of protein-protein interfaces is degenerate, close to complete, and highly connected. Proc Natl Acad Sci USA 2010; 107: 22517-22.

[106] Keskin O, Gursoy A, Ma B, Nussinov R. Principles of proteinprotein interactions: what are the preferred ways for proteins to interact? Chem Rev 2008; 108: 1225-44.

[107] Martin J. Beauty is in the eye of the beholder: proteins can recognize binding sites of homologous proteins in more than one way. PLoS Comput Biol 2010; 6: e1000821.

[108] Clackson T, Wells JA. A hot spot of binding energy in a hormonereceptor interface. Science 1995; 267: 383-6.

[109] Bogan AA, Thorn KS. Anatomy of hot spots in protein interfaces. J Mol Biol 1998; 280: 1-9.

[110] Shulman-Peleg A, Shatsky M, Nussinov R, Wolfson HJ. Spatial chemical conservation of hot spot interactions in protein-protein complexes. BMC Biol 2007; 5: 43.

[111] Aytuna AS, Gursoy A, Keskin O. Prediction of protein-protein interactions by combining structure and sequence conservation in protein interfaces. Bioinformatics 2005; 21: 2850-5.

[112] Moreira IS, Fernandes PA, Ramos MJ. Hot spots--a review of the protein-protein interface determinant amino-acid residues. Proteins 2007; 68: 803-12.

[113] Hu Z, Ma B, Wolfson H, Nussinov R. Conservation of polar residues as hot spots at protein interfaces. Proteins 2000; 39: 33142

[114] Ahmad S, Keskin O, Mizuguchi K, Sarai A, Nussinov R. CCRXP exploring clusters of conserved residues in protein structures Nucleic Acids Res 2010; 38: W398-401.

[115] Halperin I, Wolfson H, Nussinov R. Protein-protein interactions; coupling of structurally conserved residues and of hot spots across interfaces. Implications for docking. Structure 2004; 12: 1027-38.

[116] Gao Y, Wang R, Lai L. Structure-based method for analyzing protein-protein interfaces. J Mol Model 2004; 10: 44-54.

[117] Guerois R, Nielsen JE, Serrano L. Predicting changes in the stability of proteins and protein complexes: a study of more than 1000 mutations. J Mol Biol 2002; 320: 369-87.

[118] Huo S, Massova I, Kollman PA. Computational alanine scanning of the 1:1 human growth hormone-receptor complex. J Comput Chem 2002; 23: 15-27.

[119] Rajamani D, Thiel S, Vajda S, Camacho CJ. Anchor residues in protein-protein interactions. Proc Natl Acad Sci USA 2004; 101 11287-92.

[120] Moreira IS, Fernandes PA, Ramos MJ. Hot spot occlusion from bulk water: a comprehensive study of the complex between the lysozyme HEL and the antibody FVD1.3. J Phys Chem B 2007; 111: 2697-706.

[121] Guney E, Tuncbag N, Keskin O, Gursoy A. HotSprint: database of computational hot spots in protein interfaces. Nucleic Acids Res 2008; 36: D662-6.

[122] Ofran Y, Rost B. Protein-protein interaction hotspots carved into sequences. PLoS Comput Biol 2007; 3: e119.

[123] Acuner Ozbabacan SE, Gursoy A, Keskin O, Nussinov R. Conformational ensembles, signal transduction and residue ho spots: application to drug discovery. Curr Opin Drug Discov Devel 2010; 13: 527-37.

[124] Brenke R, Kozakov D, Chuang GY, et al. Fragment-based identification of druggable 'hot spots' of proteins using Fourier domain correlation techniques. Bioinformatics 2009; 25: 621-7.

[125] Ma B, Nussinov R. Trp/Met/Phe hot spots in protein-protein interactions: potential targets in drug design. Curr Top Med Chem 2007; 7: 999-1005.

[126] Ma B, Pan Y, Gunasekaran K, et al. The contribution of the Trp/Met/Phe residues to physical interactions of p53 with cellular proteins. Phys Biol 2005; 2: S56-66.

[127] Wells JA, McClendon CL. Reaching for high-hanging fruit in drug discovery at protein-protein interfaces. Nature 2007; 450: 1001-9.

[128] Ideker T, Sharan R. Protein networks in disease. Genome Res 2008; 18: 644-52. 
[129] Kann MG. Protein interactions and disease: computational approaches to uncover the etiology of diseases. Brief Bioinform 2007; 8: 333-46

[130] Wachi S, Yoneda K, Wu R. Interactome-transcriptome analysis reveals the high centrality of genes differentially expressed in lung cancer tissues. Bioinformatics 2005; 21 : 4205-8.

[131] Jonsson PF, Bates PA. Global topological features of cancer proteins in the human interactome. Bioinformatics 2006; 22: 22917.

[132] Lim J, Hao T, Shaw C, et al. A protein-protein interaction network for human inherited ataxias and disorders of Purkinje cell degeneration. Cell 2006; 125: 801-14.

[133] Amberger J, Bocchini CA, Scott AF, Hamosh A. McKusick's Online Mendelian Inheritance in Man (OMIM). Nucleic Acids Res 2009; 37: D793-6.

[134] $\mathrm{Xu} \mathrm{J,} \mathrm{Li} \mathrm{Y.} \mathrm{Discovering} \mathrm{disease-genes} \mathrm{by} \mathrm{topological} \mathrm{features} \mathrm{in}$ human protein-protein interaction network. Bioinformatics 2006; 22: 2800-5.

[135] Goh KI, Cusick ME, Valle D, et al. The human disease network. Proc Natl Acad Sci USA 2007; 104: 8685-90.

[136] Kitano H. Biological robustness. Nat Rev Genet 2004; 5: 826-37.

[137] Hase T, Tanaka H, Suzuki Y, Nakagawa S, Kitano H. Structure of protein interaction networks and their implications on drug design. PLoS Comput Biol 2009; 5: e1000550.

[138] Zhu M, Gao L, Li X, et al. The analysis of the drug-targets based on the topological properties in the human protein-protein interaction network. J Drug Target 2009; 17: 524-32.

[139] Xenarios I, Salwinski L, Duan XJ, et al. DIP, the Database of Interacting Proteins: a research tool for studying cellular networks of protein interactions. Nucleic Acids Res 2002; 30: 303-5

[140] Isserlin R, El-Badrawi RA, Badery GD. The Biomolecular Interaction Network Database in PSI-MI 2.5. Database. The Journal of Biological Databases and Curation 2011.

[141] Stark C, Breitkreutz BJ, Reguly T, et al. BioGRID: a general repository for interaction datasets. Nucleic Acids Res 2006; 34 : D535-9.

[142] Hermjakob H, Montecchi-Palazzi L, Lewington C, et al. IntAct: an open source molecular interaction database. Nucleic Acids Res 2004; 32: D452-5.

[143] Peri S, Navarro JD, Kristiansen TZ, et al. Human protein reference database as a discovery resource for proteomics. Nucleic Acids Res 2004; 32: D497-501

[144] Schoof H, Spannagl M, Yang L, et al. Munich information center for protein sequences plant genome resources: a framework for integrative and comparative analyses 1(W). Plant Physiol 2005; 138: 1301-9.

[145] Han K, Park B, Kim H, Hong J, Park J. HPID: the Human Protein Interaction Database. Bioinformatics 2004; 20: 2466-70.

[146] Gong S, Yoon G, Jang I, et al. PSIbase: a database of Protein Structural Interactome map (PSIMAP). Bioinformatics 2005; 21: 2541-3.

[147] Raghavachari B, Tasneem A, Przytycka TM, Jothi R. DOMINE: a database of protein domain interactions. Nucleic Acids Res 2008; 36: D656-61.

[148] Aloy P, Russell RB. Interrogating protein interaction networks through structural biology. Proc Natl Acad Sci USA 2002; 99: 5896-901.

[149] Dawelbait G, Winter C, Zhang Y, et al. Structural templates predict novel protein interactions and targets from pancreas tumour gene expression data. Bioinformatics 2007; 23: i1 15-24.

[150] Huang YJ, Hang D, Lu LJ, et al. Targeting the human cancer pathway protein interaction network by structural genomics. Mol Cell Proteomics 2008; 7: 2048-60.
[151] Kar G, Gursoy A, Keskin O. Human cancer protein-protein interaction network: a structural perspective. PLoS Comput Biol 2009; 5: e1000601.

[152] Tuncbag N, Gursoy A, Nussinov R, Keskin O. Predicting proteinprotein interactions on a proteome scale by matching evolutionary and structural similarities at interfaces using PRISM. Nat Protoc 2011; 6: 1341-54.

[153] Gursoy A, Keskin O, Nussinov R. Topological properties of protein interaction networks from a structural perspective. Biochem Soc Trans 2008; 36: 1398-403.

[154] Tuncbag N, Kar G, Gursoy A, Keskin O, Nussinov R. Towards inferring time dimensionality in protein-protein interaction networks by integrating structures: the p53 example. Mol Biosyst 2009; 5: 1770-8.

[155] Keskin O, Gursoy A, Ma B, Nussinov R. Towards drugs targeting multiple proteins in a systems biology approach. Curr Top Med Chem 2007; 7: 943-51.

[156] Sugaya N, Ikeda K, Tashiro T, et al. An integrative in silico approach for discovering candidates for drug-targetable proteinprotein interactions in interactome data. BMC Pharmacol 2007; 7 10.

[157] Aloy P, Bottcher B, Ceulemans H, et al. Structure-based assembly of protein complexes in yeast. Science 2004; 303: 2026-9.

[158] Xie L, Bourne PE. Structure-based systems biology for analyzing off-target binding. Curr Opin Struct Biol 2011; 21: 189-99.

[159] Chong CR, Sullivan DJ, Jr. New uses for old drugs. Nature 2007; 448: 645-6.

[160] DiMasi JA, Hansen RW, Grabowski HG. The price of innovation: new estimates of drug development costs. J Health Econ 2003; 22 151-85.

[161] Kinnings SL, Liu N, Buchmeier N, et al. Drug discovery using chemical systems biology: repositioning the safe medicine Comtan to treat multi-drug and extensively drug resistant tuberculosis. PLoS Comput Biol 2009; 5: e1000423.

[162] Weber A, Casini A, Heine A, et al. Unexpected nanomolar inhibition of carbonic anhydrase by COX-2-selective celecoxib: new pharmacological opportunities due to related binding site recognition. J Med Chem 2004; 47: 550-7.

[163] Frantz S. Drug discovery: playing dirty. Nature 2005; 437: 942-3.

[164] Paolini GV, Shapland RH, van Hoorn WP, Mason JS, Hopkins AL. Global mapping of pharmacological space. Nat Biotechnol 2006; 24: 805-15.

[165] Lee SJ, Wang JY. Exploiting the promiscuity of imatinib. J Biol 2009; 8: 30 .

[166] Druker BJ. Translation of the Philadelphia chromosome into therapy for CML. Blood 2008; 112: 4808-17.

[167] Winger JA, Hantschel O, Superti-Furga G, Kuriyan J. The structure of the leukemia drug imatinib bound to human quinone reductase 2 (NQO2). BMC Struct Biol 2009; 9: 7.

[168] Nagar B, Bornmann WG, Pellicena P, et al. Crystal structures of the kinase domain of $\mathrm{c}-\mathrm{Abl}$ in complex with the small molecule inhibitors PD173955 and imatinib (STI-571). Cancer Res 2002; 62: 4236-43.

[169] Kanehisa M. The KEGG database. Novartis Found Symp 2002; 247: 91-101; discussion -3, 19-28, 244-52

[170] Xie L, Bourne PE. Functional coverage of the human genome by existing structures, structural genomics targets, and homology models. PLoS Comput Biol 2005; 1: e31.

[171] Tuncbag N, Keskin O, Gursoy A. HotPoint: hot spot prediction server for protein interfaces. Nucleic Acids Res 2010; 38: W402-6. 